\title{
Experimental Validation of a Novel Extracellular-Based Source Representation of Acute Myocardial Ischemia
}

\author{
Brian Zenger ${ }^{1,2,3,4}$, Jake A Bergquist ${ }^{1,2,3}$, Wilson W Good ${ }^{1,2,3}$, Lindsay C Rupp ${ }^{1,2,3}$, Rob S \\ MacLeod $^{1,2,3}$ \\ ${ }^{1}$ Scientific Computing and Imaging Institute, University of Utah, SLC, UT, USA \\ ${ }^{2}$ Nora Eccles Cardiovascular Research and Training Institute, University of Utah, SLC, UT, USA \\ ${ }^{3}$ Department of Biomedical Engineering, University of Utah, SLC, UT, USA \\ ${ }^{4}$ School of Medicine, University of Utah, SLC, UT, USA
}

\begin{abstract}
Electrocardiographic imaging (ECGI) based detection of myocardial ischemia requires an accurate formulation of the source model, which includes a relationship between extracellular and transmembrane potentials (TMPs). In this study, we used high-resolution intramural experimental recordings and forward modeling to examine the relationship between extracellular potentials and TMPs during myocardial ischemia. We measured extracellular electrograms from intramural plunge needle arrays during seven controlled ischemia episodes in an animal model. We used three TMP source representations: (1) parameterized and distance-based (defined previously), (2) extracellularbased linear transform, and (3) extracellular-based sigmoidal transform. TMPs for each source formulation were then used to compute extracellular potentials by calculating the passive bidomain forward solution throughout the myocardium. We compared measured and computed potentials. Linear and sigmoidal approaches produced improved results compared to the parameterized method. The RMSE, SC, and TC of linear, sigmoidal, and parameterized methods were $0.85 \mathrm{mV}, 1.21 \mathrm{mV}$, and $3.37 \mathrm{mV} ; 0.91$, 0.88 , and $0.47 ; 0.90,0.77$, and 0.33 respectively. We found extracellular-based calculations of TMPs produced superior forward computations compared to parameterized zones.
\end{abstract}

\section{Introduction}

Acute myocardial ischemia is a highly complex pathophysiological event that develops from reduced myocardial perfusion. This reduced perfusion leads to a deficit in necessary nutrients and a build-up of toxic metabolites within the cardiac tissue. These pathological changes alter cardiac electrical activity and increase the likelihood of cardiac arrhythmias or sudden cardiac death.[1] The patho- logical cellular alterations that occur during ischemia are well understood; however, tissue and whole-heart level ischemic development is complex, multifaceted, and difficult to predict. [2]

To improve our understanding of ischemic development at the whole-heart level, computational models have been developed that solve forward problems of electrocardiography. These solutions can compute ischemic bioelectric potentials within the heart, on the heart surface, or over the torso surface using conductivity values, geometric models, and a representation of the ischemic source. Our recent studies have used one such approach with experimentally derived ischemic zones to compute ischemic potentials on the heart and torso surfaces. [3, 4] These methods use the passive bidomain formulation of the forward problem of ischemia, in which the ischemic source captures known alterations in transmembrane potentials (TMP's), parameterized with a threshold-distance based approach from experimentally recorded extracellular potentials. The threshold-distance approach emulates ischemic zones by thresholding extracellular potentials recorded during the ST segment to identify ischemic cores. The transition zone between the ischemic core and healthy tissue is then modeled using a distance-based decay of TMP amplitude. The threshold-distance parameterization assumes all myocardial ischemia has such a central core with extracellular potentials during the ST segment that exceed an absolute threshold value; it also assumes that the transition zone extends isotropically a set distance from the ischemic core independent of location, anatomy, or cardiac fiber orientation. These assumptions produce models that ignore key features about acute myocardial ischemia and may fail to capture the temporal and spatial complexity seen experimentally, which limits the predictive accuracy of the model.

To improve the accuracy of forward computed ischemic potentials, we developed two novel extracellular-based pa- 
rameterizations, which we designed to capture the spatial and temporal complexity of acute ischemia throughout its development. We identified two transfer functions to assign a TMP value for extracellular potential values recorded nearby. To validate our method, we compared the measured and forward computed intramural potentials using the threshold-distance and the novel extracellular TMP parameterization methods. We found a significant spatial and temporal improvement in computed potentials using the novel extracellular-based parameterizations.

\section{Methods}

Forward Problem: We modeled myocardial ischemia using the passive bidomain formulation described previously.[5, 6] Briefly, this method uses transmembrane potentials $\left(\Phi_{m}\right)$ as the source and solves for extracellular potentials $\left(\Phi_{e}\right)$ throughout the myocardium with the equation

$$
\nabla \cdot\left(\sigma_{i} \nabla \Phi_{m}\right)=-\nabla \cdot\left(\sigma_{h} \nabla \Phi_{e}\right),
$$

where $\sigma_{e}$ is extracellular conductivity, $\sigma_{i}$ is intracellular conductivity, and $\sigma_{h}=\sigma_{e}+\sigma_{i}$ with $\sigma_{h}$ representing bulk myocardial conductivity. Conductivity tensors were constructed from measured principal fiber directions and previously determined conductivity ratios. [3, 4]

TMP Parameterizations: TMP values $\left(\Phi_{m}\right)$ were parameterized using both the threshold-distance and novel extracellular approaches. In short, the threshold-distance approach used extracellular ST40\% potential threshold of $4.5 \mathrm{mV}$ to isolate ischemic cores from recorded extracellular potentials within the myocardium. The difference between ischemic and healthy tissue TMP's was set to $35 \mathrm{mV}$. A distance-based decay of TMP's was applied based on a piecewise function from the ischemic core boundary to $11 \mathrm{~mm}$ into the myocardium (figure 1 panel A.). [7] The novel extracellar approach did not assume any spatial variation but rather an explicit dependence of drop in transmembrane potential to measured extracellular potential at the ST segment. We specifically applied both linear and sigmoid transfer functions to relate these values, as shown in figure 1 panel B.

Experimental Dataset: The measured electrograms were acquired using a method described previously.[8]. Briefly, an in situ pig heart was instrumented with 20 intramural plunge needle recording arrays, each with ten electrodes spaced $1.8 \mathrm{~mm}$ apart along the shaft of the needle. Electrodes were sampled simultaneously using a digital multiplexer at $1 \mathrm{kHz}$. Resulting unipolar signals were filtered, baseline corrected, and fiducialized using the opensource PFEIFER software.[9]

Controlled acute myocardial ischemia was induced via decreasing perfusion and increasing myocardial stress. Perfusion was reduced by $90 \%$ using a surgically placed
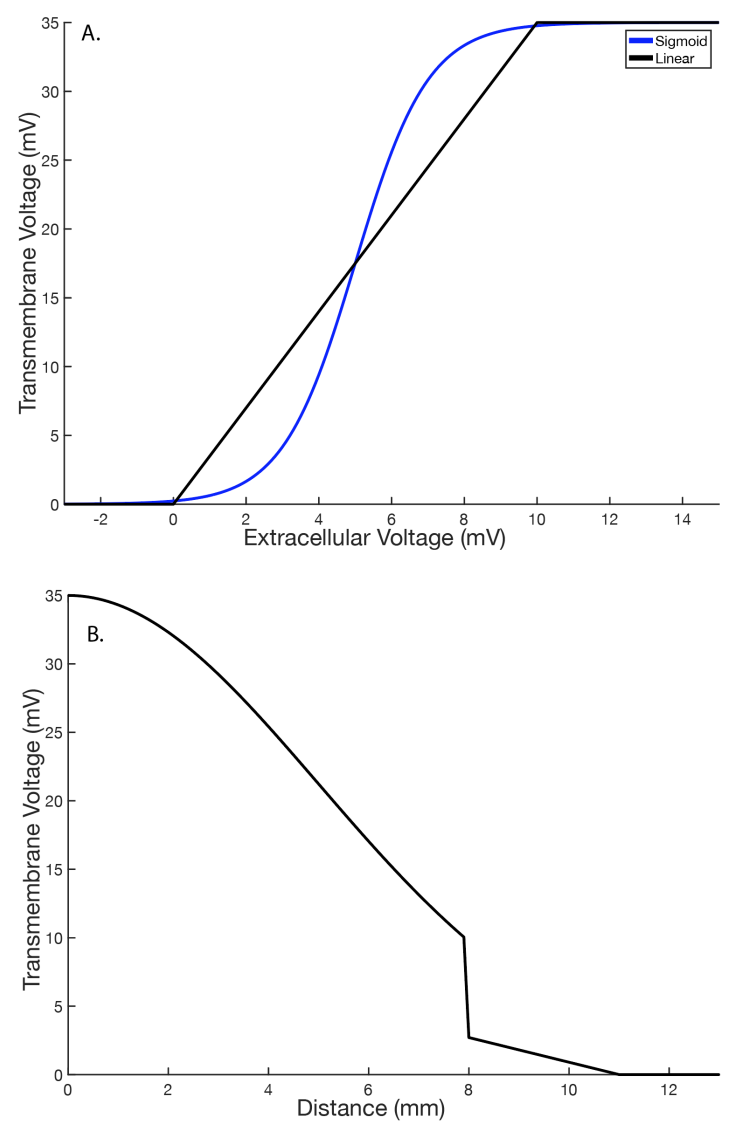

Figure 1. Ischemic changes in TMP ampitude. A. Transfer function between the measured extracellular potential during the ST segment and transmembrane potentials (in $\mathrm{mV}$ ) B. Distance based approach to reduced TMP amplitude during ischemia

hydraulic occluder around the left anterior descending coronary artery (LAD). Myocardial stress was induced using electrical pacing and pharmacological stimulation to mimic a clinical cardiac stress tests. We identified ischemia from changes to the measured potentials $40 \%$ into the ST segment.

The geometric models were constructed from highresolution magnetic resonance imaging (MRI) of the explanted, fixed heart. Myocardial structures and needle electrode locations were segmented from the MRI using the Seg3d open-source software www.seg3d.org and meshed using the Cleaver software. Principle fiber directions were calculated from separate MRI scans using diffusion tensor imaging (DTI).

Evaluation Metrics: Metrics included spatial correlation (SC), temporal correlation (TC), and root-meansquare error (RMSE) to assess differences between measured and forward-computed needle electrode extracellular potentials, as described previously.[10] The temporal 
correlation examines the correlation between the signals averaged across all electrodes. The spatial correlation examines the correlation averaged across all time instances.

\section{Results}

ST40\% potentials were measured and computed across seven individual episodes of acute myocardial ischemia from 400 selected heart beats. Forward solutions were computed from TMPs parameterized with each of the proposed methods (threshold-distance, extracellular linear, and extracellular sigmoid based), and compared on the intramural needle electrode nodes. Figure 2 shows an example of measured vs. forward computed potentials using all three methods. As shown, the threshold-distance approach produced large spherical regions with isotropic decay of ischemic to healthy tissue over a set distance. Extracellular linear has significant variation, matching the measured potentials. From the statistics across all measurement nodes and times in table 1, both linear and sigmoid approaches significantly improved the correlation and RMSE metrics of the threshold distance approach. The linear method performed the best, with a spatial correlation of 0.91 , temporal correlation of 0.90 , and RMSE of $0.85 \mathrm{mV}$. The Thresholddistance-based method performed the worst with a spatial correlation of 0.47 , temporal correlation of 0.33 , and an RMSE of $3.37 \mathrm{mV}$.

Table 1. Global performance metrics of temporal correlation (TC), spatial correlation (SC), and root mean squared error (RMSE) (mV) across all ischemic episodes.

\begin{tabular}{cccc} 
& Threshold-distance & Linear & Sigmoid \\
\hline \hline RMSE & 3.37 & 0.85 & 1.21 \\
SC & 0.47 & 0.91 & 0.88 \\
TC & 0.33 & 0.90 & 0.77 \\
\hline \hline
\end{tabular}

\section{Discussion}

We hypothesized that the novel extracellular-based TMP parameterization would produce more accurate forward computed ischemic potentials than the current thresholddistance-based approaches. The results based on experimental recordings confirmed our hypothesis and showed significant increases - up to 0.5-in the temporal and spatial correlation values and a significant decrease in RMSE of over one $\mathrm{mV}$ using extracellular-based approaches compared to the threshold-distance approach. We found relatively small differences between the linear and sigmoidal transfer functions, which we consider negligible compared to the significant improvements over the thresholddistance approach. Using an extracellular-based parameterization highlights the need for a more sophisticated ap- proach to parameterize TMPs and improve the calculation of ischemic potentials.

In this study we observed complex spatial patterns of ischemic zones that were not reproduced well by the threshold-distance based parameterization. The thresholddistance approach seeks to simplify parameterization of ischemia by assuming an ischemic core region surrounded by a small transition zone based on a set distance from the core boundary. This method created large spherical zones throughout the myocardium, which did not match the measured ischemic potentials. Specifically we found significant amounts of moderately ischemic tissue without central cores and in spatially complex patterns not captured by setting a binary threshold. Our findings show that during their development, ischemic zones do not have 'central cores' and will be misidentified as 'normal' by the threshold-distance-based parameterizations.

We also note the threshold-distance-based decay applied isotropically from the ischemic core boundary is a fundamentally questionable assumption. The combination of cellular connectivity and myocardial fiber direction makes it unlikely that the transition region between healthy and ischemic tissue is dependent only on myocardial structures and anatomy. Figure 2 shows the anisotropic nature of the transition zone depending on the anatomical location. Future projects will explore which anatomical variables are essential to capture this anisotropic development.

Another key observation was the dynamic temporal ischemic development. Conventional approaches have focused on computing potentials during severe ischemia. In this study, we applied our parameterization approach from the start to the end of multiple graded ischemic episodes. We found the threshold-distance parameterization produced low temporal correlation values, especially early in the interventions. Specifically, we showed the temporal performance of the threshold-distance method to be 0.33 throughout all ischemic episodes. Using the extracellularpotential-based approach, we correctly identified 'intermediate' ischemia throughout an ischemic episode with temporal correlations above 0.80 . This observation highlights the dynamic nature of ischemia and the complexity introduced during transient cardiac stress.

This study has some limitations. We used an experimental model which may not mimic human physiology and ischemia development perfectly. Additionally, we had limited access to place plunge needle arrays and prioritized sampling in the presumed perfusion bed. [11]

This study lays the groundwork for several important future projects. First, our modified source representation of ischemia in the forward model can be used as a building block to improve the localization of ischemia from body surface electrodes and the inverse model, i.e., electrocardiographic imaging. Better non-invasive detection of is- 

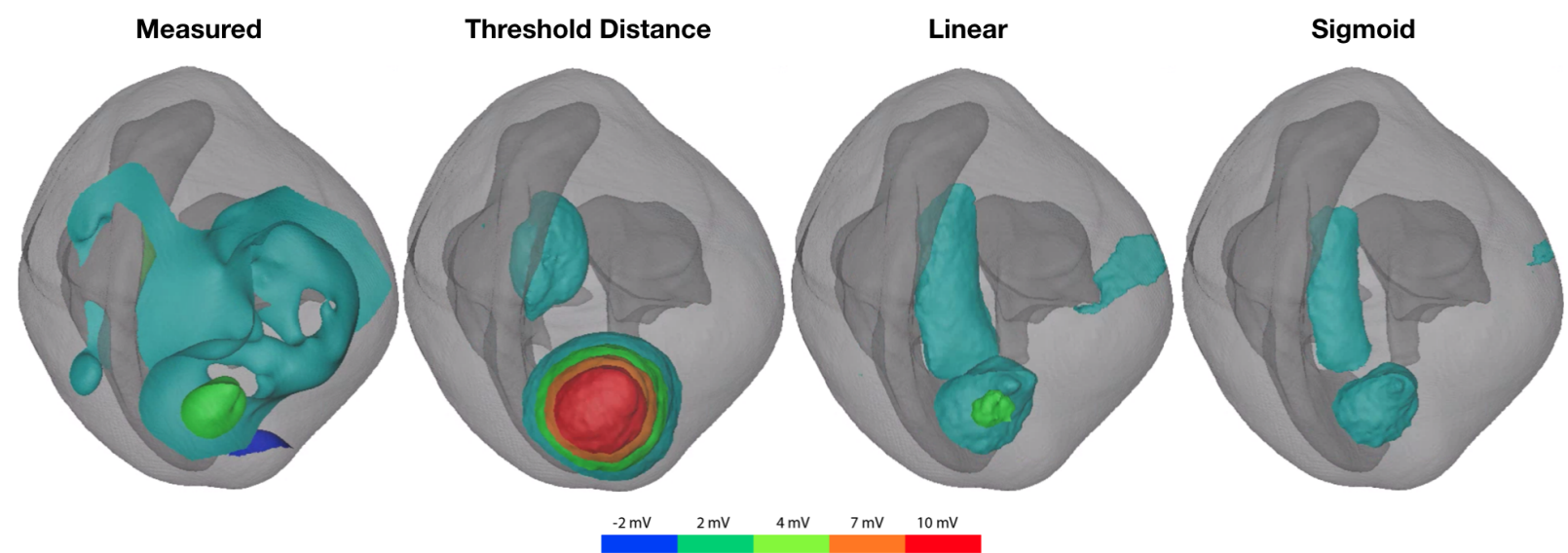

Figure 2. Example extracellular measured and computed ischemic potentials based on all three parameterization methods. Measurements were taken early in the ischemic episode, when only small amounts of ischemia was present.

chemia would enable more accurate patient risk stratification and help guide treatment. Our results also highlight the critical transient and dynamic physiological features of acute myocardial ischemia and the need to carefully examine ischemia using multiple modeling, experimental, and clinical approaches. Additionally, our results will contribute to ongoing projects attempting to simulate acute myocardial ischemia in patient-specific modeling applications, which could also be used for risk stratification, diagnosis, and treatment planning.

\section{Acknowledgments}

Support for this research came from NIH NIGMS grant no. P41GM103545, NIH NHLBI Fellowship grant no. 1F30HL149327-01A1, and the Nora Eccles Treadwell Foundation for Cardiovascular Research.

\section{References}

[1] Stern S. State of the art in stress testing and ischaemia monitoring. Cardiac Electrophysiology Review 2002;6(3):204208.

[2] Li D, Li C, Yong A, Kilpatrick D. Source of electrocardiographic ST changes in subendocardial ischemia. Circ Res 1998;82:957-970.

[3] Burton B. Subject-Specific Modeling of Partial Thickness Cardiac Ischemia: From Experiment to Body Surface. Ph.D. thesis, University of Utah, 2018.

[4] Burton B, Aras K, Good W, Tate J, Zenger B, MacLeod R. Image-based modeling of acute myocardial ischemia using experimentally derived ischemic zone source representations. J Electrocardiol 2018;51(4):725-733.

[5] Burstein B, Nattel S. Atrial structural remodeling as an antiarrhythmic target. J Cardiovasc Pharmacol Jul 2008; 52(1):4-10.

[6] Burstein B, Nattel S. Atrial fibrosis: mechanisms and clin- ical relevance in atrial fibrillation. J Am Coll Cardiol Feb 2008;51(8):802-809.

[7] Swenson D, Stinstra J, Burton B, Aras K, Healy L, , MacLeod R. Evaluating the effects of border zone approximations with subject specific ischemia models. In Doessel O, Schlegel WC (eds.), World Congress on Med. Phys. and Biomed. Eng., volume 25/IV. Heidelberg: Springer, 2009; 1680-1683.

[8] Zenger B, Good WW, Bergquist JA, Burton BM, Tate JD, Berkenbile L, Sharma V, MacLeod RS. Novel experimental model for studying the spatiotemporal electrical signature of acute myocardial ischemia: a translational platform. Physiological Measurement 2020;41(1):15002. ISSN 13616579.

[9] Rodenhauser A, Good W, Zenger B, Tate J, Aras K, Burton B, MacLeod R. PFEIFER: Preprocessing framework for electrograms intermittently fiducialized from experimental recordings. J Open Source Software 2018;3(21):472.

[10] Bergquist J, Good W, Zenger B, Tate J, MacLeod R. Optimizing the reconstruction of cardiac potentials using a novel high resolution pericardiac cage. In Computing in Cardiology 2019, volume 46. 2019; 1-4.

[11] Bhuiya FA, Pitts SR, McCaig LF. Emergency department visits for chest pain and abdominal pain: United States, 1999-2008. NCHS data brief September 2010;(43):18. ISSN 1941-4935. URL http: // europepmc.org/ abstract/MED/20854746

Address for correspondence:

Brian Zenger

brian.zenger@hsc.utah.edu 